\title{
Glucose mediates insulin sensitivity via a hepatoportal mechanism in high-fat-fed rats
}

\author{
Holly M Johnson'1, Erin Stanfield ${ }^{1}$, Grace J Campbell'1, Erica E Eberl'1, Gregory J Cooney² and Kim S Bell-Anderson ${ }^{1}$ \\ 1The University of Sydney, Charles Perkins Centre, School of Life and Environmental Sciences, Sydney, New South Wales, Australia \\ ${ }^{2}$ The University of Sydney, Charles Perkins Centre, School of Medical Sciences, Sydney, New South Wales, Australia
}

Correspondence should be addressed to K S Bell-Anderson: kim.bellanderson@sydney.edu.au

\begin{abstract}
Poor nutrition plays a fundamental role in the development of insulin resistance, an underlying characteristic of type 2 diabetes. We have previously shown that high-fat dietinduced insulin resistance in rats can be ameliorated by a single glucose meal, but the mechanisms for this observation remain unresolved. To determine if this phenomenon is mediated by gut or hepatoportal factors, male Wistar rats were fed a high-fat diet for 3 weeks before receiving one of five interventions: high-fat meal, glucose gavage, highglucose meal, systemic glucose infusion or portal glucose infusion. Insulin sensitivity was assessed the following day in conscious animals by a hyperinsulinaemic-euglycaemic clamp. An oral glucose load consistently improved insulin sensitivity in high-fat-fed rats, establishing the reproducibility of this model. A systemic infusion of a glucose load did not affect insulin sensitivity, indicating that the physiological response to oral glucose was not due solely to increased glucose turnover or withdrawal of dietary lipid. A portal infusion of glucose produced the largest improvement in insulin sensitivity, implicating a role for the hepatoportal region rather than the gastrointestinal tract in mediating the effect of glucose to improve lipid-induced insulin resistance. These results further deepen our understanding of the mechanism of glucose-mediated regulation of insulin sensitivity and provide new insight into the role of nutrition in whole body metabolism.
\end{abstract}

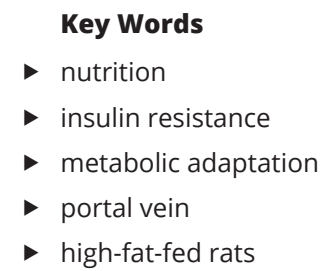

Journal of Endocrinology (2019) 241, 189-199

\section{Introduction}

The increasing prevalence of type 2 diabetes mellitus (T2DM) parallels the relentless increase in obesity across the globe. The International Diabetes Federation predicts a further increase of $55 \%$ in the incidence of T2DM worldwide by 2035 (International Diabetes Federation 2013). Insulin resistance is considered a primary pathology in the development of T2DM. How and why the body fails to respond to insulin is not fully understood but overnutrition and macronutrient composition likely play a role in the regulation of the underlying mechanisms (Parillo \& Riccardi 2004). There is much controversy around the optimal diet for maintenance of insulin action and there is lack of a clear consensus on macronutrient subtype recommendations to maintain metabolic health (Daly et al. 1997, McClenaghan 2005, Feinman et al. 2015). Elucidation of mechanisms linking nutrients to regulation of insulin action is critical for improving current treatments of T2DM as diet modification is almost always prescribed and is easily manipulated.

Increased energy intake from either fat or carbohydrate, resulting in a positive energy balance, produces insulin resistance in rodents and humans (Small et al. 2018). High-fat feeding in rats causes profound whole-body and tissue-specific insulin resistance and is a routinely used means of inducing metabolic dysfunction in rodent models. In 1987, Storlien et al. clearly showed 
that under isocaloric conditions, dietary fat subtypes exert varying effects on insulin sensitivity measured under hyperinsulinaemic-euglycaemic clamp conditions (Storlien et al. 1987). Much research has been fat-focused and concentrated on the association of ectopic lipid with the development of insulin resistance in the chronic setting, but the mechanisms and causal role of lipids in insulin resistance remain unclear (Buettner et al. 2006, Turner et al. 2013). There is no question that nutrition plays a fundamental role in modulating metabolism and insulin action. However, little is currently known about how specific nutrients regulate insulin sensitivity acutely.

We previously reported that substituting one highglucose meal for the usual high-fat meal in high-fat-fed rats restored insulin sensitivity when determined the following day by direct measurement using hyperinsulinaemiceuglycaemic clamps (Oakes et al. 1997). This work also revealed a negative correlation between muscle glucose uptake and the concentration of lipid intermediates in muscle, but failed to isolate the mechanism underlying the improvement in insulin sensitivity in response to a single high-glucose meal.

Typically, when energy contribution from carbohydrate is increased in the diet, total fat is decreased (as protein content is maintained), and vice versa: when fat is increased, the carbohydrate contribution is necessarily reduced. It is likely that the quantity and types of dietary carbohydrate, fat and protein are important for determining insulin action both in the short and long term. A question remaining to be answered from our prior observations was whether the acute increase in insulin action caused by a single glucose meal is due to the glucose per se or a reduction in fat in the diet. Further, if glucose is responsible, where does the signal to muscle to enhance insulin-stimulated glucose disposal originate?

In the experiments described here, we determined that the physiological adaptive increase in insulin sensitivity in response to glucose was dependent on delivery via the oral route and unlikely to be due to lipid withdrawal. Further, glucose infusion into the portal vein was even more effective in enhancing whole-body insulin action, suggesting a role for the hepatoportal region in adaptive regulation of insulin action in response to incoming nutrients.

\section{Materials and methods}

\section{Animals, diets and interventions}

Three separate experiments were performed. All rats were adult male Wistars obtained from the Animal
Resource Centre (ARC Perth, Australia). All experiments were conducted in accordance with protocols approved by either the Garvan Institute Animal Experimentation Ethics Committee (Study 1) or the University of Sydney Animal Care and Ethics Committee (Studies 2 and 3). Rats were housed on a $12 \mathrm{~h}$ light:darkness cycle in a temperature-controlled room $\left(22 \pm 1{ }^{\circ} \mathrm{C}\right)$ in groups of two to four until surgery and individually post surgery. All rats were given 1 week to acclimatise and fed irradiated chow (Specialty Feeds, WA, Australia) before the commencement of studies. The first study was conducted in the animal facilities at the Garvan Institute. Studies 2 and 3 were conducted at the University of Sydney.

\section{Study 1}

Forty-three rats weighing approximately $200 \mathrm{~g}$ were fed either standard laboratory chow ad libitum or fixed rations of a high-fat diet for 3 weeks $(350 \mathrm{~kJ} /$ day $)$. The chow diet was composed of $21 \%$ protein, $7 \%$ fat and $72 \%$ carbohydrate expressed as energy, with an energy density of $14 \mathrm{MJ} / \mathrm{kg}$ (Rat and Mouse Cubes, Norco Stockfeeds, NSW, Australia). The high-fat diet was $21 \%$ protein, $59 \%$ fat (mainly as safflower oil) and 20\% carbohydrate $(24 \mathrm{MJ} / \mathrm{kg}$, composed in-house). One week before experiments, rats were fitted with carotid and jugular cannulae to enable blood sampling and infusion during the clamps. Rats were anaesthetised with a mixture of ketamine hydrochloride (Ketalar, $60 \mathrm{mg} / \mathrm{kg}$, Parke-Davis Pty. Ltd., NSW, Australia) and xylazine (Rompun, $10 \mathrm{mg} / \mathrm{kg}$, Bayer Australia Ltd., NSW, Australia) injected intraperitoneally. Rats were monitored daily post surgery for this study and subsequent studies. On the eve before clamp experiments, a subset of high-fat-fed rats were gavaged with $3 \mathrm{~mL}$ of $55 \%$ glucose solution twice $(16: 00 \mathrm{~h}$ and $20: 00 \mathrm{~h} ; \sim 60 \mathrm{~kJ})$ instead of receiving their usual high-fat meal. A hyperinsulinaemiceuglycaemic clamp was performed on rats the following morning (see below). Rats were killed with an intravenous bolus of sodium pentobarbital $(40 \mathrm{mg} / \mathrm{kg})$.

\section{Study 2}

To test if glucose can also improve insulin action in dietinduced obesity, 46 rats weighing 200-250g were fed either chow or high-fat diet ad libitum for 3 weeks. The chow diet was irradiated and composed of $23 \%$ protein, $12 \%$ fat and $65 \%$ carbohydrate as energy $(14 \mathrm{MJ} / \mathrm{kg}$; Specialty Feeds Rat and Mouse Cubes, Glen Forrest, WA, Australia). The high-fat diet was $22 \%$ protein, $46 \%$ fat (mainly as lard) and 32\% carbohydrate $(21.8 \mathrm{MJ} / \mathrm{kg}$, composed in-house). All rats underwent surgery at 2 weeks to place dual jugular vein cannulas. 
Rats were initially anaesthetised in an induction chamber with $4 \%$ isoflurane supplied in medical grade oxygen at $0.4 \mathrm{~L} / \mathrm{min}$ (Stinger apparatus, Advanced Anaesthesia Specialists, NSW, Australia). Rats were maintained on $2 \%$ isoflurane at $0.2 \mathrm{~L} / \mathrm{min}$ via a nosecone during surgery. On the eve before clamp experiments, high-fat-fed rats were subset into three groups: high-fat-fed rats, high-fat-fed rats given a single high-glucose meal and high-fat-fed rats infused over $2 \mathrm{~h}$ with a $50 \%$ glucose solution via a jugular cannula. The high-glucose meal was isocaloric with the high-fat meal and composed of $21.5 \%$ protein, $9.5 \%$ fat and $69 \%$ carbohydrate as glucose and cornstarch $(16 \mathrm{MJ} / \mathrm{kg}$, composed in-house). The infused glucose solution $(\sim 78 \mathrm{~kJ})$, contained an equivalent amount of glucose as the glucose meal. Insulin sensitivity was assessed the next morning with a hyperinsulinaemic-euglycaemic clamp after which rats were killed with an intravenous bolus of sodium pentobarbital ( $40 \mathrm{mg} / \mathrm{kg}$ of body weight). Tissues were rapidly excised in order: red quadriceps muscle, epididymal WAT and intrascapular BAT. Tissues were snap frozen in liquid nitrogen and stored at $-80^{\circ} \mathrm{C}$.

\section{Study 3}

Forty rats weighing 140-160g were fed a high-fat diet (430 kJ/day provided, same composition as in Study 2) for 3 weeks. All rats underwent surgery at 2 weeks to place a portal vein and dual jugular vein cannulas. Surgery was performed under anaesthesia as in Study 2. On the eve before clamp experiments, rats were subset into four groups: high-fat-fed rats, high-fat-fed rats given a single high-glucose meal (same composition as in Study 2), high-fat-fed rats infused over $4 \mathrm{~h}$ with a $50 \%$ glucose solution $(\sim 78 \mathrm{~kJ})$ via a jugular cannula or high-fat-fed rats infused similarly via the portal cannula. A subset of rats were placed in individual Promethion metabolic cages (Promethion High Definition Multiplexed Respirometry System for rats, Sable Systems International, NV, USA) for two nights, with sampling every $5 \mathrm{~min}$, for determination of energy expenditure by indirect calorimetry and calculation of respiratory quotient (RQ: ratio of oxygen consumed to carbon dioxide expelled). Because these cages are not sealed, and instead rely on pulled air flow engineering, we were able to perform glucose infusions and swap meals without interrupting gas measurements. Insulin sensitivity was assessed the next morning with a hyperinsulinaemic-euglycaemic clamp after which rats were killed as in Study 2. Tissues were rapidly excised in order: red quadriceps muscle, white quadriceps muscle, liver, epididymal WAT and intrascapular BAT. Tissues were stored as in Study 2.

(C) 2019 Society for Endocrinology Published by Bioscientifica Ltd. Printed in Great Britain

\section{Hyperinsulinaemic-euglycaemic clamps}

Animals were fasted for $5 \mathrm{~h}$ before the commencement of clamp experiments (Kraegen et al. 1985). Insulin was infused at a constant rate of $0.25 \mathrm{IU} / \mathrm{kg} /$ hour and $25 \%$ glucose in ultrapure water (Arium Pro VF, Sartorius Stedium Australia Pty Ltd, Dandenong South, VIC, Australia) was infused at a variable rate. Small blood samples $(\sim 50 \mu \mathrm{L})$ were taken at five- to ten-minute intervals and blood glucose concentration determined with an Accu-Chek Performa glucometer (Roche Diabetes Care). Blood glucose levels continued to be monitored and the rate of the glucose infusion adjusted accordingly to achieve a stable blood glucose concentration. The glucose infusion rate (GIR) is a direct measure of insulin sensitivity and, along with peripheral glucose disposal (Rd) and liver glucose output (Ra), was determined as described (Kraegen et al. 1985).

Rats were infused with a mix of radiolabelled D-glucose and 2-Deoxy-D glucose (2DG) as a bolus to the rat via a jugular cannulae. Blood samples $(200 \mu \mathrm{L})$ were taken at 2, 5, 10, 15, 20, 30 (and 45 in Studies 2 and 3) min and plasma added to $100 \mathrm{mM} \mathrm{ZnSO} 4$ to which an equal volume of $50 \mathrm{mM} \mathrm{BaOH}$ was added for measurement of plasma radioactivity in a scintillation counter (Tri-Carb 2810TR, Perkin Elmer).

\section{Plasma biochemistry}

Plasma was collected in heparin and frozen at $-20^{\circ} \mathrm{C}$ until required. Plasma insulin concentration was determined by radioimmunoassay (RIA) (Sensitive Rat Insulin RIA kit, SRI-13K, Merck Millipore). Plasma triglyceride concentration was determined using an automated analyser (AU480, Beckman Coulter) and triglyceride reagent (OSR60118, Beckman Coulter).

\section{Tissue glucose metabolic index and triglyceride content}

Snap-frozen liver and muscle tissues were ground in a mortar and pestle with liquid nitrogen to create a powder. The tissue powder was used in all subsequent analyses. Tissue samples were homogenised in chloroform: methanol (2:1) solution using a bead mill (Mini-Beadbeater 24, Biospec Products Inc. OK, USA). $0.6 \%(\mathrm{w} / \mathrm{v})$ sodium chloride solution was added and the homogenate vigorously vortexed, and then centrifuged $\left(956 \mathrm{~g}, 10 \mathrm{~min}, 23^{\circ} \mathrm{C}\right)$ to separate the aqueous and organic phases, which were used for determination of $\mathrm{Rg}^{\prime}$ and triglyceride respectively.

For $\mathrm{Rg}^{\prime}$ measurements, an aliquot of the aqueous phase was passed through an anion exchange resin column 
(AG 1-X8, hydroxide form, Bio-Rad Laboratories Pty Ltd) to bind phosphorylated 2DG in the sample and the flow through collected (representing unphosphorylated 2DG). A second aliquot was pipetted directly into a separate scintillation vial and ultrapure water added (total 2DG). The amount of phosphorylated 2DG was calculated as the difference between the two samples (total - free).

For measurement of triglyceride, red muscle and liver samples were homogenised as above, with the addition of 2-h incubation at room temperature prior to the addition of sodium chloride solution. The organic phase was collected into a fresh polypropylene tube and dried under nitrogen. The resulting pellet was resuspended in 100\% ethanol and analysed for triglyceride as above.

\section{Statistical analysis}

All data in tables are presented as mean \pm S.E.M. Significant differences were determined by ANOVA followed by pairwise comparisons by $t$-tests with pooled S.D. using the Bonferroni method of adjustment for multiple comparisons. When samples were paired, differences were determined by paired $t$-test. Differences were considered statistically significant at $P<0.05$. R software (R Core Team 2016) was used for all statistical analysis and data visualisation.

\section{Results}

We performed a series of incremental experiments in rats to investigate the underlying mechanism responsible for glucose-induced improvement in whole-body insulin sensitivity. Changes in insulin sensitivity were measured in high-fat-fed rats given a glucose gavage, a glucose meal or infusions of glucose systemically or portally to help locate the site of action by differentiating the routes of glucose delivery.

\section{Insulin sensitivity in high-fat-fed rats is improved by glucose gavage}

The first question we asked was whether glucose by itself was able to ameliorate insulin resistance in the high-fatfed rat. Rats were fed isocaloric diets of chow or high fat (60\% fat by energy as safflower oil) for 3 weeks and then a subgroup was gavaged with glucose instead of their usual high-fat meal. Insulin sensitivity was assessed the next day ( $14 \mathrm{~h}$ later) via hyperinsulinaemic-euglycaemic clamps.

Body weight of chow versus fat rats was not different, as expected, when fed the same amount of calories $(350 \mathrm{~kJ} /$ day). However, high-fat-fed rats receiving the glucose gavage only received $123 \mathrm{~kJ}$ which was equivalent to amounts of glucose given in a meal previously and shown to be effective in reducing insulin resistance (Burcelin et al. 2000, Coate et al. 2013) (Fig. 1A).

Rats only receiving high-fat food had significantly greater basal blood glucose levels compared with chow-fed rats and glucose gavage had little effect (Fig. 1B) despite significantly lowering basal insulin levels (Fig. 1C). Highfat-fed rats were insulin resistant compared with chow-fed rats as determined by the GIR during the hyperglycaemiceuglycaemic clamp. Glucose gavage substantially improved insulin sensitivity in the high-fat-fed rats (Fig. 1D). There were no significant differences in plasma glucose or insulin between the groups at the end of the clamp (Table 1).

\section{Systemic delivery of glucose does not improve insulin sensitivity in high-fat-fed rats}

We next asked, can we also improve insulin sensitivity by infusion of glucose over several hours into the jugular vein, simulating the rise in blood glucose in a fat-fed rat

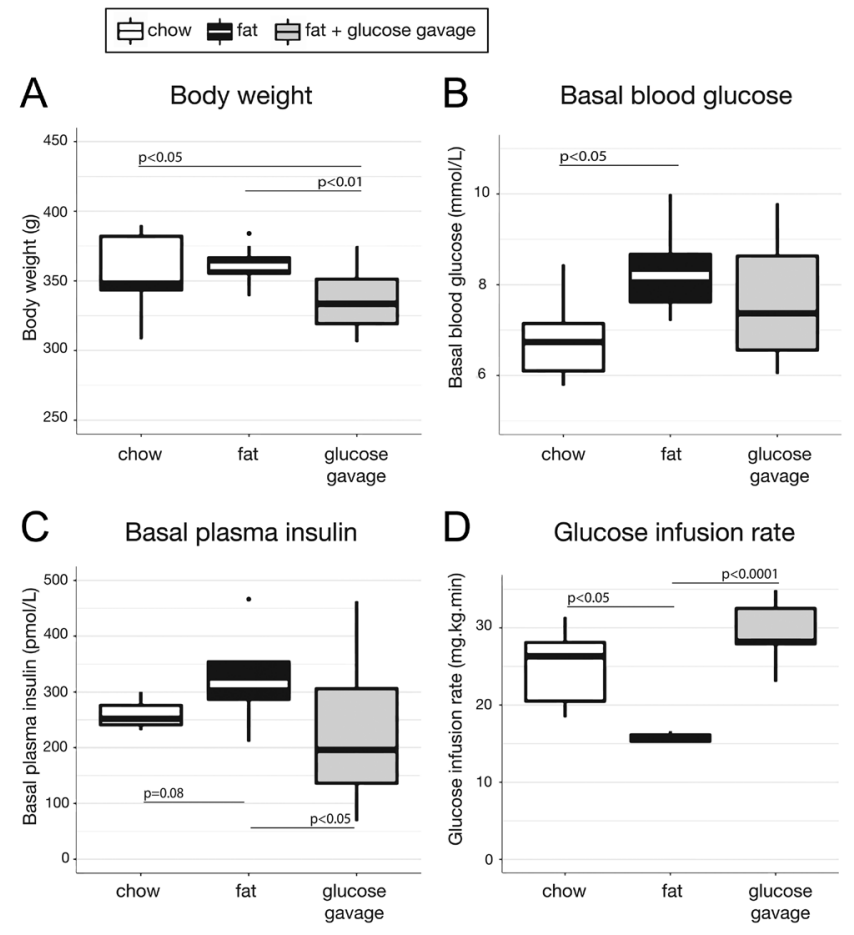

\section{Figure 1}

(A) Body weight of rats fed either chow $(n=11)$, high-fat diet $(n=12)$ or high-fat diet + glucose gavage $(n=20)$. (B) Basal blood glucose levels in rats fed either chow $(n=7)$, high-fat diet $(n=13)$ or high-fat diet + glucose gavage $(n=18)$. (C) Basal plasma insulin levels in rats fed either chow $(n=9)$, high-fat diet $(n=12)$ or high-fat diet + glucose gavage $(n=19)$.

(D) Glucose infusion rate during a 30-min hyperinsulinaemic-euglycaemic clamp in rats fed either chow $(n=5)$, high-fat diet $(n=5)$ or high-fat diet + glucose gavage $(n=10)$. Data are mean \pm S.E.M. 
Table 1 Plasma glucose and insulin levels during a 30-min hyperinsulinaemic-euglycaemic clamp in rats fed either chow $(n=5)$, high-fat diet $(n=5)$ or high-fat diet + glucose gavage $(n=10)$.

\begin{tabular}{|c|c|c|c|}
\hline & Chow & Fat & $\begin{array}{c}\text { Fat + glucose } \\
\text { gavage }\end{array}$ \\
\hline $\begin{array}{l}\text { Clamp plasma } \\
\text { glucose (mmol/L) }\end{array}$ & $6.5 \pm 0.4$ & $7.6 \pm 0.4$ & $7.9 \pm 0.4$ \\
\hline $\begin{array}{l}\text { Clamp plasma } \\
\text { insulin (pmol/L) }\end{array}$ & $957 \pm 111$ & $986 \pm 85$ & $741 \pm 116$ \\
\hline
\end{tabular}

Data are mean \pm S.E.M. No significant differences were detected.

receiving glucose orally. We also optimised experimental conditions such that the composition of the fat diet changed to a lard-based diet with added sucrose $(45 \%$ energy from fat, mainly lard), more reflective of 'Western diet' macronutrient composition. To further extend relevance to humans, rats were allowed to consume diets ad libitum and were older, and therefore heavier.

Given the strong relationship between fat and insulin resistance, it was appropriate to consider whether the improvement in insulin sensitivity in our model was related to the withdrawal of lipid from the diet. In addition to the groups, chow, high-fat and high-fat plus a single high-glucose meal, we included a high-fat-fed group that received an intravenous infusion of glucose calorically equivalent to the high-glucose meal (after adjusting for absorption by the gastrointestinal tract) and delivered over $4 \mathrm{~h}$ in the evening before the clamp studies.

At clamp, after 3 weeks, ad libitum high-fat feeding, all high-fat-fed groups were significantly heavier than chowfed rats (Fig. 2A) and body weight between the high-fatfed groups were not different. Basal plasma glucose levels were within the expected physiological range for all rats, however, both groups receiving glucose the evening before clamps had significantly lower basal glucose levels the following day than chow and high-fat-fed rats (Fig. 2B). High-fat feeding increased basal plasma insulin concentrations and a single high-glucose meal significantly ameliorated circulating insulin to levels comparable with chow-fed controls (Fig. 2C). As observed in Study 1, highfat feeding in rats markedly reduced insulin sensitivity compared with chow-fed rats as measured by the GIR during clamp studies (Fig. 2D). Further, a single highglucose meal significantly ameliorated this reduction in GIR. The intravenous infusion of glucose did not alter insulin resistance in the high-fat-fed rat despite them having not received their usual high-fat meal and therefore being deprived of dietary fat the evening before insulin sensitivity was assessed; thus, lipid withdrawal was not responsible for the improvement in insulin resistance.
Blood glucose levels and GIR over time are shown in Fig. 3A and B, respectively.

No significant differences in clamp plasma glucose or insulin levels was detected amongst high-fat-fed rats, however levels of both tended to be greater in high-fat-fed rats compared with chow controls regardless of glucose interventions (Table 2). Whole-body glucose disposal (Rd) was significantly increased by a single high-glucose meal in high-fat-fed rats, but no change was observed in hepatic glucose production ( $\mathrm{Ra}$ ) between groups. At the tissue level, a significant reduction in red quadriceps muscle glucose metabolic index $\left(\mathrm{Rg}^{\prime}\right)$ was observed in high-fat-fed rats and rats receiving a systemic glucose infusion compared with chow controls (Table 2).

\section{Portal infusion of glucose increases insulin sensitivity in high-fat-fed rats}

The oral versus peripheral route of glucose delivery and the difference in the effect on insulin sensitivity suggest the presence of factors triggered by the ingestion,

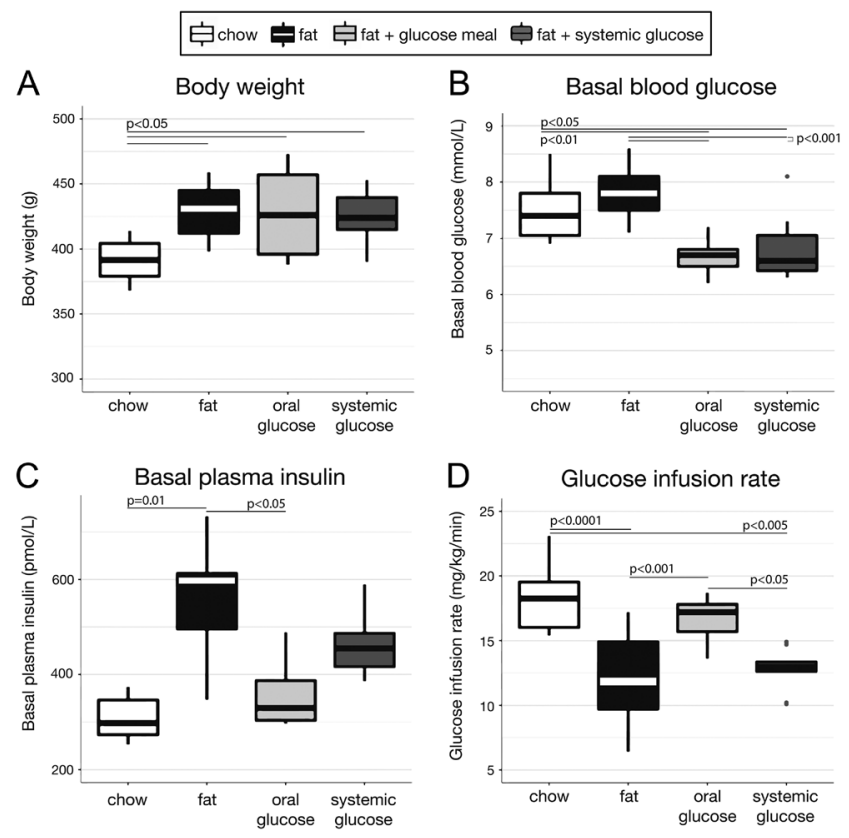

\section{Figure 2}

(A) Body weight of rats fed either chow $(n=8)$, high-fat diet $(n=13)$, high-fat diet + high-glucose meal $(n=11)$ or high-fat diet + systemic glucose infusion $(n=11)$. (B) Basal blood glucose levels in rats fed either chow $(n=7)$, high-fat diet $(n=13)$, high-fat diet + high-glucose meal $(n=9)$ or high-fat diet + systemic glucose infusion $(n=10)$. (C) Basal plasma insulin levels in rats fed either chow $(n=8)$, high-fat diet $(n=12)$, high-fat diet + high-glucose meal $(n=11)$ or high-fat diet + systemic glucose infusion ( $n=11$ ). (D) Glucose infusion rate during a hyperinsulinaemiceuglycaemic clamp in rats fed either chow $(n=8)$, high-fat diet $(n=13)$, high-fat diet + high-glucose meal $(n=11)$ or high-fat diet + systemic glucose infusion $(n=10)$. 

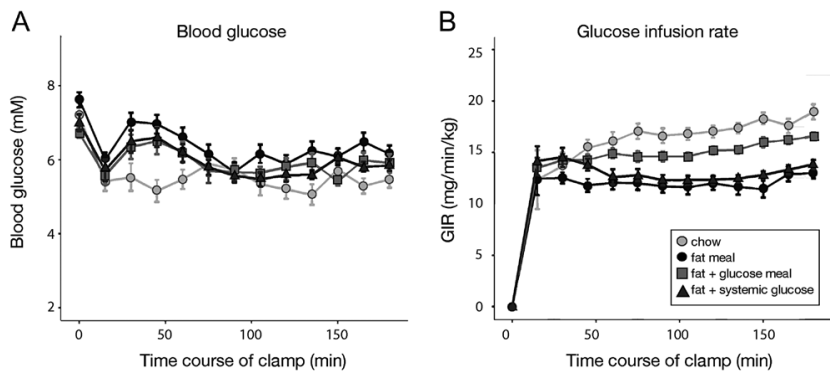

Figure 3

(A) Blood glucose levels and (B) glucose infusion rate during a hyperinsulinaemic-euglycaemic clamp in rats fed either chow $(n=8)$, high-fat diet $(n=13)$, high-fat diet + high-glucose meal $(n=11)$ or high-fat diet + systemic glucose infusion $(n=10)$.

absorption or metabolism of glucose. To further isolate the critical location of the ingested glucose signal, we performed the experiments again and included a group of high-fat-fed rats that received a glucose infusion directly into the portal vein the evening before clamp experiments.

All rats in Study 3 were high-fat-fed for 3 weeks and were of similar body weights on the morning of clamp experiments (Fig. 4A). Rats in all groups recovered well from surgery indicated by consistent weight gain post surgery during the week prior to experiments (Table 3). No significant differences were detected between groups in basal blood glucose levels or basal plasma insulin concentrations immediately prior to clamps (Fig. 4B and C). Levels of glucose and insulin during clamp experiments were not different between groups. Plasma triglyceride was sufficiently suppressed by insulin in all groups. Basal plasma triglyceride was significantly lowered by systemic infusion of glucose in the high-fat-fed rat (Table 3).

As in the previous experiments, insulin sensitivity was not altered in rats infused systemically with glucose which were as insulin resistant as high-fat-fed rats receiving their usual high-fat meal (Fig. 4D). Again, rats that consumed a high-glucose meal had a significantly higher GIR than high-fat-fed controls indicating improved insulin sensitivity. Furthermore, GIR was significantly higher in rats receiving a portal glucose infusion than in all other groups (Fig. 4D). Blood glucose levels and GIR over time are shown in Fig. 5A and $\mathrm{B}$, respectively. Calculated Rd suggests that improved insulin sensitivity is mainly due to increased uptake of glucose into tissues rather than enhanced suppression of liver glucose production (Table 3). Insulin-stimulated tissue $\mathrm{Rg}^{\prime}$ in red quadriceps muscle was significantly higher in portal glucose infused rats compared with systemic glucoseinfused rats. Surprisingly, glucose uptake into epididymal WAT was reduced in high-fat-fed rats that received a portal infusion. No significant differences were detected in liver or red quadriceps muscle triglyceride content.

To assess whether the different glucose interventions affected body composition, we measured lean mass and fat mass by MRI $24 \mathrm{~h}$ prior to the clamp and the morning of the clamp (EchoMRI 100, EchoMedical Systems, Houston, TX, USA) (Fig. 6). High-fat-fed rats consuming either a high-fat meal or high-glucose meal maintained their body weight over this period, however, while fat-fed controls displayed no changes in body composition, rats receiving a single glucose meal slightly increased lean mass and decreased fat mass. Both glucose infusion groups dropped body weight over the $24 \mathrm{~h}$ period, as expected due to the reduction in calories received. Both lean and fat mass contributed to this loss in body weight, but changes in fat mass were such that fat as a percentage of body mass was reduced and lean percentage increased.

We also measured energy expenditure and RQ in the $24 \mathrm{~h}$ leading up to clamp experiments (Fig. 7). Energy expenditure increased during the night phase in both mealfed groups to a similar extent. Both glucose infusion groups tended to have reduced energy expenditure during the night phase compared with meal-fed groups (mean nighttime energy expenditure of high-fat-fed versus systemic glucose $P=0.08$, portal glucose $P=0.051$; oral glucose versus

Table 2 Plasma glucose, insulin levels and glucose disposal ( $R d)$, production $(R a)$ and metabolic index (Rg') in red quadriceps muscle, epididymal WAT or BAT during a hyperinsulinaemic-euglycaemic clamp in rats fed either chow $(n=7-8)$, high-fat diet $(n=11)$, high-fat diet + glucose meal $(n=8-11)$ or high-fat diet + systemic glucose infusion $(n=8-10)$.

Clamp plasma glucose $(\mathrm{mmol} / \mathrm{L})$
Clamp plasma insulin $(\mathrm{pmol} / \mathrm{L})$
$\mathrm{Rd}(\mathrm{mg} / \mathrm{kg} / \mathrm{min})$
$\mathrm{Ra}(\mathrm{mg} / \mathrm{kg} / \mathrm{min})$
Muscle $\mathrm{Rg}(\mu \mathrm{mol} / 100 \mathrm{~g} / \mathrm{min})$
Epididymal WAT $\mathrm{Rg}(\mu \mathrm{mol} / 100 \mathrm{~g} / \mathrm{min})$
BAT $\mathrm{Rg}^{\prime}(\mu \mathrm{mol} / 100 \mathrm{~g} / \mathrm{min})$

\begin{tabular}{c}
\hline Chow \\
\hline $6.7 \pm 0.3$ \\
$666 \pm 67$ \\
$23.5 \pm 1.4$ \\
$5.2 \pm 1.4$ \\
$33.9 \pm 6.1$ \\
$1.8 \pm 0.6$ \\
$129 \pm 25$
\end{tabular}

\begin{tabular}{c}
\hline Fat \\
\hline $7.9 \pm 0.2$ \\
$907 \pm 42$ \\
$19.9 \pm 1.4$ \\
$7.7 \pm 1.3$ \\
$21.5 \pm 2.7$ \\
$0.6 \pm 0.1$ \\
$106 \pm 13$ \\
\hline
\end{tabular}

\begin{tabular}{c}
\hline Fat + glucose meal \\
\hline $7.7 \pm 0.3$ \\
$992 \pm 71$ \\
$25.5 \pm 0.9 \#$ \\
$9.0 \pm 1.2$ \\
$24.5 \pm 3.0$ \\
$0.5 \pm 0.1$ \\
$132 \pm 19$ \\
\hline
\end{tabular}

Fat + systemic glucose

$7.5 \pm 0.1$

$1038 \pm 84$

$22.5 \pm 1.8$

$9.4 \pm 1.7$

$10.8 \pm 2.1^{*}$

$0.7 \pm 0.1$

$84 \pm 9$

Data are mean \pm S.E.M.

$\star P<0.05, \# P<0.05$ versus high fat. 


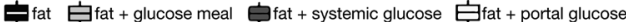

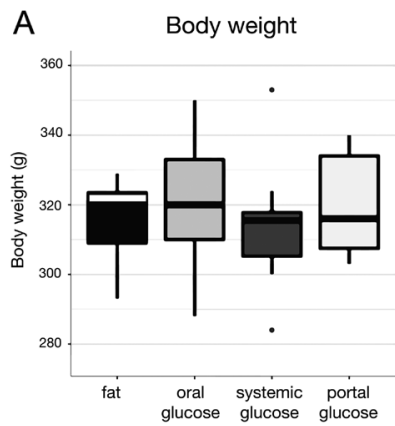

B Basal blood glucose
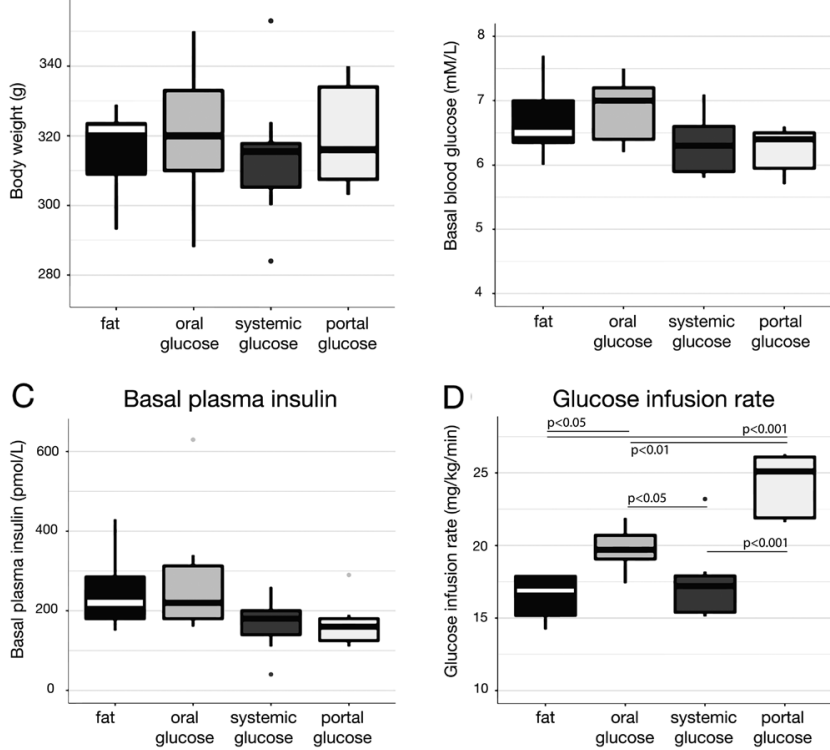

Figure 4

(A) Body weight of rats fed either high-fat diet $(n=8)$, high-fat diet + highglucose meal $(n=9)$, high-fat diet + systemic glucose infusion $(n=10)$ or high-fat diet + portal glucose infusion $(n=11)$. (B) Basal blood glucose levels in rats fed either high-fat diet $(n=7)$, high-fat diet + high-glucose meal $(n=9)$, high-fat diet + systemic glucose infusion $(n=9)$ or high-fat diet + portal glucose infusion $(n=11)$. (C) Basal plasma insulin levels in rats fed either high-fat diet $(n=7)$, high-fat diet + high-glucose meal $(n=6)$, high-fat diet + systemic glucose infusion $(n=6)$ or high-fat diet + portal glucose infusion ( $n=7$ ). (D) Glucose infusion rate during a hyperinsulinaemic-euglycaemic clamp in rats fed either high-fat diet $(n=6)$, high-fat diet + high-glucose meal $(n=6)$, high-fat diet + systemic glucose infusion $(n=6)$ or high-fat diet + portal glucose infusion $(n=5)$.

portal glucose $P=0.084$ ), likely reflecting absence of energy expenditure relating to the thermic effect of food and as adaptation to the reduced incoming calories. The RQ which represents whole-body fuel type utilisation reflected the interventions the rats received. RQ increased significantly in all rats receiving glucose, indicating a switch from oxidation of fat to carbohydrate. Interestingly, a glucose infusion rapidly elevated and maintained RQ at a level of 1 , during the four-hour period of infusion, before plummeting to mainly fat oxidation on cessation of glucose (mean nighttime RQ of high-fat-fed versus systemic glucose $P<0.01$, portal glucose $P<0.01$; oral glucose versus systemic glucose $P<0.001$, portal glucose $P<0.001$ ).

\section{Discussion}

In the current series of experiments, we investigated the importance of route of delivery of glucose to the observation that a single glucose meal can improve insulin action, as measured by the hyperinsulinaemic-euglycaemic clamp, in high-fat-fed rats. Increases in insulin sensitivity in response to glucose were evident with oral delivery and are likely due to enhanced glucose disposal rather than suppression of liver glucose production. We show that the improvement in insulin action in high-fat-fed rats is not due to lipid withdrawal, as fat-fed rats receiving glucose systemically remain insulin resistant. In contrast, glucose infusion directly into the portal vein was very effective in enhancing insulin-stimulated glucose disposal, suggesting a role for portal glucosensors in the adaptive regulation of insulin action in response to incoming nutrients.

We determined insulin sensitivity directly using the hyperinsulinaemic-euglycaemic clamp technique in two experiments where we used radioisotopes to determine the contribution of liver and peripheral tissues to whole body insulin action. We observed significant differences in insulin sensitivity as directly determined by the GIR in high-fat-fed rats. We measured a reduction in insulinstimulated glucose uptake into red muscle of high-fat-fed rats compared with chow controls, and further reduction in rats receiving glucose systemically. Intriguingly, portal infusion of glucose suppressed visceral WAT glucose uptake, suggesting that portal glucose insulin-stimulated glucose disposal is selectively enhanced in target tissues. Glucose uptake into other insulin-dependent and independent tissues, such as red and mixed skeletal muscle, subcutaneous WAT and heart, will help better determine the effect of ingested or portal glucose on glucose utilisation. We did not detect changes in liver glucose production $(\mathrm{Ra})$, but there was significant experimental error associated with our tracer measurements. Constant infusion of tracer may be more accurate in determining Ra directly by tracer dilution (Vella \& Rizza 2009).

Other studies performing portal glucose infusions in rodents report effects during infusion and have led to the identification of glucose sensors in the portal vein itself and connected to the brain via afferent hepatic branches of the vagus nerve (Berthoud et al. 1992). The portal vein drains visceral adipose tissue and the intestine, and carries blood rich in nutrients, metabolites and factors derived from the diet, microbiota and adipose to the liver. As such, it is a potentially key anatomical point where incoming nutrients and metabolites, along with gut-derived hormones, are sensed and this information relayed to the brain to prepare the body for disposal of ingested energy. Glucose infusion into the portal vein in mice is enough to induce hypoglycaemia and increase glucose utilisation by oxidative tissues (muscle and BAT, but also heart) 
Table 3 Plasma glucose, insulin levels and glucose disposal (Rd), production ( $R a)$ and metabolic index (Rg') during a hyperinsulinaemic-euglycaemic clamp in rats fed either high-fat diet ( $n=6-7)$, high-fat diet + high-glucose meal ( $n=5-7)$, high-fat diet + systemic glucose infusion $(n=5-9)$ or high-fat diet + portal glucose infusion $(n=5-9)$.

Weight gain post surgery $(\mathrm{g})$
Clamp plasma glucose $(\mathrm{mmol} / \mathrm{L})$
Clamp plasma insulin $(\mathrm{pmol} / \mathrm{L})$
Basal plasma triglyceride $(\mathrm{mmol} / \mathrm{L})$
Clamp plasma triglyceride $(\mathrm{mmol} / \mathrm{L})$
$\mathrm{Rd}(\mathrm{mg} / \mathrm{kg} / \mathrm{min})$
$\mathrm{Ra}(\mathrm{mg} / \mathrm{kg} / \mathrm{min})$
$\mathrm{Red} \mathrm{muscle} \mathrm{Rg}^{\prime}(\mu \mathrm{mol} / 100 \mathrm{~g} / \mathrm{min})$
White muscle $\mathrm{Rg}^{\prime}(\mu \mathrm{mol} / 100 \mathrm{~g} / \mathrm{min})$
Epididymal WAT $\mathrm{Rg}(\mu \mathrm{mol} / 100 \mathrm{~g} / \mathrm{min})$
BAT Rg' $(\mu \mathrm{mol} / 100 \mathrm{~g} / \mathrm{min})$
Liver triglyceride $(\mu \mathrm{mol} / \mathrm{mg})$
Muscle triglyceride $(\mu \mathrm{mol} / \mathrm{mg})$

\begin{tabular}{c}
\hline Fat \\
\hline $34.7 \pm 4.6$ \\
$9.0 \pm 0.2$ \\
$502 \pm 33$ \\
$1.8 \pm 0.1$ \\
$0.8 \pm 0.04$ \\
$23.2 \pm 1.7$ \\
$8.5 \pm 0.9$ \\
$20.6 \pm 1.8$ \\
$4.6 \pm 0.8$ \\
$2.1 \pm 0.3$ \\
$106 \pm 14$ \\
$12.3 \pm 1.1$ \\
$7.0 \pm 0.7$
\end{tabular}

\begin{tabular}{c}
\hline Fat + glucose meal \\
\hline $30.9 \pm 6.3$ \\
$9.4 \pm 0.1$ \\
$645 \pm 101$ \\
$1.4 \pm 0.2$ \\
$0.6 \pm 0.03^{\#}$ \\
$27.9 \pm 3.3$ \\
$8.2 \pm 3.4$ \\
$21.4 \pm 2.3$ \\
$3.7 \pm 0.6$ \\
$1.8 \pm 0.3$ \\
$125 \pm 25$ \\
$11.5 \pm 0.9$ \\
$7.6 \pm 0.6$
\end{tabular}

\begin{tabular}{c}
\hline Fat + systemic glucose \\
\hline $35.0 \pm 2.6$ \\
$8.8 \pm 0.3$ \\
$394 \pm 31$ \\
$1.1 \pm 0.1^{\#}$ \\
$0.4 \pm 0.04^{\# \#}$ \\
$21.9 \pm 1.1^{*}$ \\
$6.6 \pm 0.8$ \\
$13.5 \pm 1.9^{*}$ \\
$1.9 \pm 0.2$ \\
$1.5 \pm 0.05$ \\
$114 \pm 18$ \\
$14.6 \pm 1.2$ \\
$6.1 \pm 0.1$ \\
\hline
\end{tabular}

\begin{tabular}{c}
\hline Fat + portal glucose \\
\hline $37.4 \pm 5.0$ \\
$8.8 \pm 0.2$ \\
$430 \pm 100$ \\
$1.3 \pm 0.1$ \\
$0.5 \pm 0.06 \# \#$ \\
$35.2 \pm 1.3^{\#}$ \\
$8.4 \pm 2.5$ \\
$26.3 \pm 3.1$ \\
$3.9 \pm 0.4$ \\
$1.1 \pm 0.1 \#$ \\
$103 \pm 14$ \\
$11.7 \pm 0.7$ \\
$7.2 \pm 0.4$
\end{tabular}

Data are mean \pm S.E.M.

$\# P<0.05, \# \# P<0.001$ versus high fat; $* P<0.05$ versus high-fat + portal glucose infusion.

(Burcelin et al. 2000). Physiological effects of glucose sensing in the portal vein include induction of satiety (Trung et al. 2014), increase hepatic glucose uptake (Coate et al. 2013) and reduction of counter-regulatory hormones induced by peripheral hypoglycaemia (Bohland et al. 2014). Blocking hepatoportal glucose sensor signalling, via surgical denervation of the portal vein, results in reduced whole body insulin sensitivity and increased blood glucose levels in mice (Trung et al. 2014). However, we are not aware of any studies reporting latent effects of portal glucose infusion as we do here. It is possible that the body regulates metabolism on a day-to-day basis and creates a 'meal memory' to optimise metabolic processes in line with the macronutrient composition of the previous meal, in anticipation of the following meal. Early evidence of acute dietary effects on glucose metabolism is reported in male subjects consuming
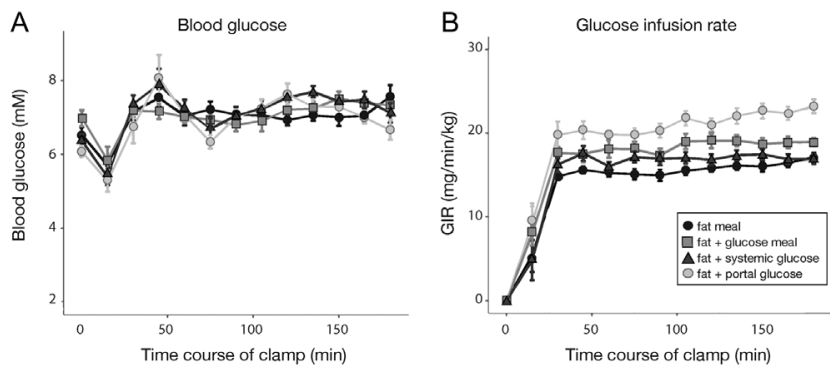

Figure 5

(A) Blood glucose levels and (B) glucose infusion rate during a hyperinsulinaemic-euglycaemic clamp in rats fed either high-fat diet $(n=6)$, high-fat diet + high-glucose meal $(n=6)$, high-fat diet + systemic glucose infusion $(n=6)$ or high-fat diet + portal glucose infusion $(n=5)$.

(C) 2019 Society for Endocrinology Published by Bioscientifica Ltd. Printed in Great Britain high-fat or carbohydrate for 2 days in a cross-over design. Consumption of high-carbohydrate diet significantly improved glucose tolerance compared with consumption of a high-fat diet (Sweeney 1927). More recently, a single oral ingestion of saturated fat in young men was shown to increase liver insulin resistance (Hernandez et al. 2017).

A negative portoarterial glucose gradient may also play an important role in letting the brain know of absence of glucose entering the body and shutting down glucose uptake in non-essential tissues. That starvation lowers glucose tolerance was first shown by Claude Bernard in 1877 (Bernard 1877). In both Study 2 and 3, we observed consistent effects of systemic glucose infusion on suppression of insulin-stimulated glucose disposal and glucose uptake into red muscle relative to the portally infused group. This portal gluco-sensing system may help the body differentiate between glucose from food and endogenously produced glucose to ensure appropriate regulation of metabolic processes in peripheral tissues in response to the fed versus the fasted state.

Our experiments also highlight the lability of the high-fat diet-induced insulin resistance and raise concern over whether this diet rodent model is appropriate for investigating the molecular determinants of insulin resistance in humans, especially if insulin resistance is characterised by a breakdown of resilience mechanisms that maintain metabolic flexibility (Goodpaster \& Sparks 2017). In Study 3 we observed that high-fat-fed rats receiving a glucose infusion were able to rapidly increase RQ for maximal carbohydrate contribution to oxidation, providing no evidence that the ability of metabolic tissues 
A

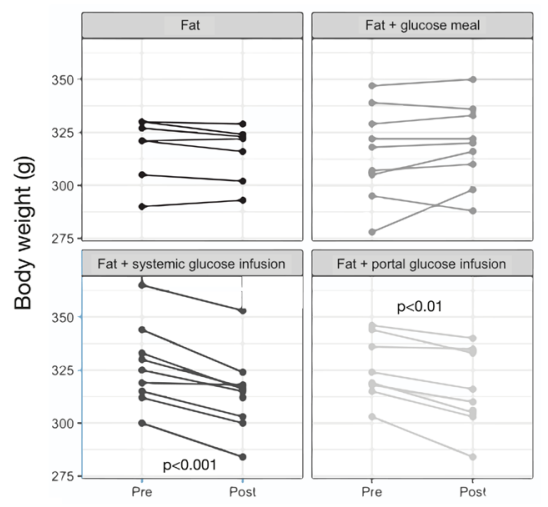

C

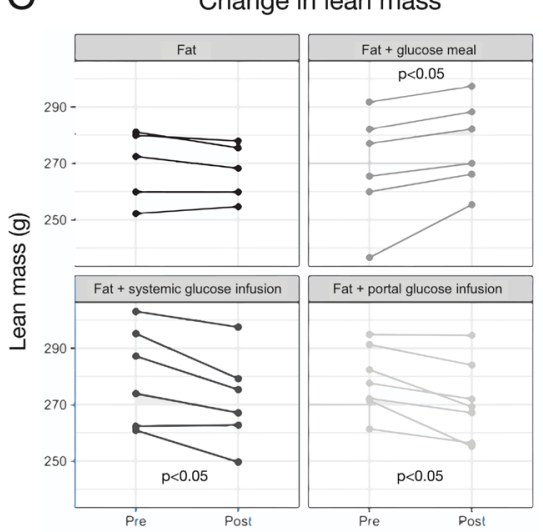

B

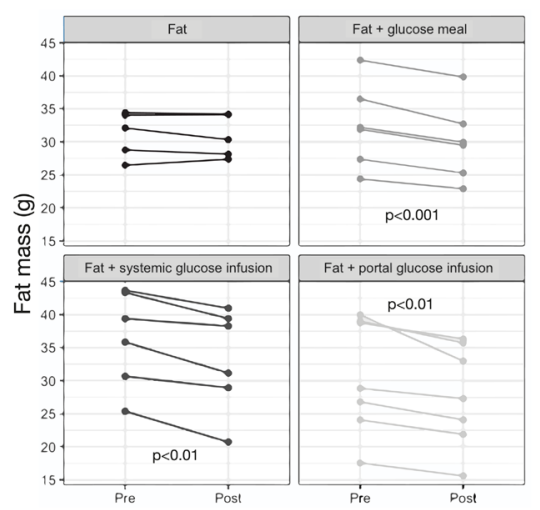

D Change in percentage of

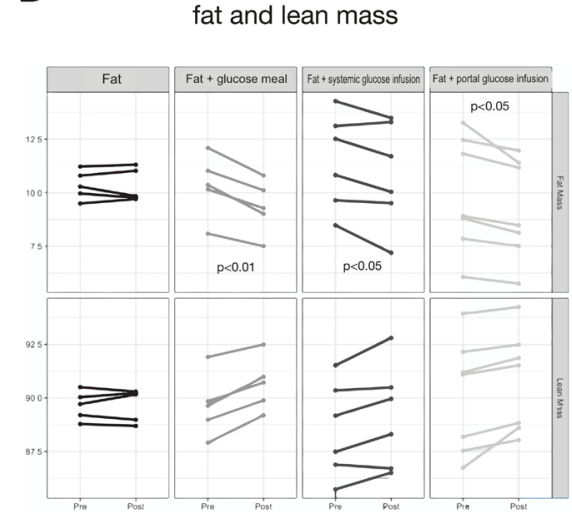

Figure 6

Comparison of (A) body weight, (B) fat mass, (C) lean mass and (D) fat and lean mass as a percentage of tissue mass before (Pre) and after (Post) glucose intervention in rats fed either high-fat diet, high-fat diet + high-glucose meal (light blue), high-fat diet + systemic glucose infusion (orange) or high-fat diet + portal glucose infusion. Rats were measured $24 \mathrm{~h}$ prior to, and on the morning of, clamp experiments. to switch between fuels and match energy expenditure to energy intake was impaired in 3-week high-fat-fed rats. A longer period of high-fat feeding may be necessary as impairment of metabolic flexibility in isolated skeletal

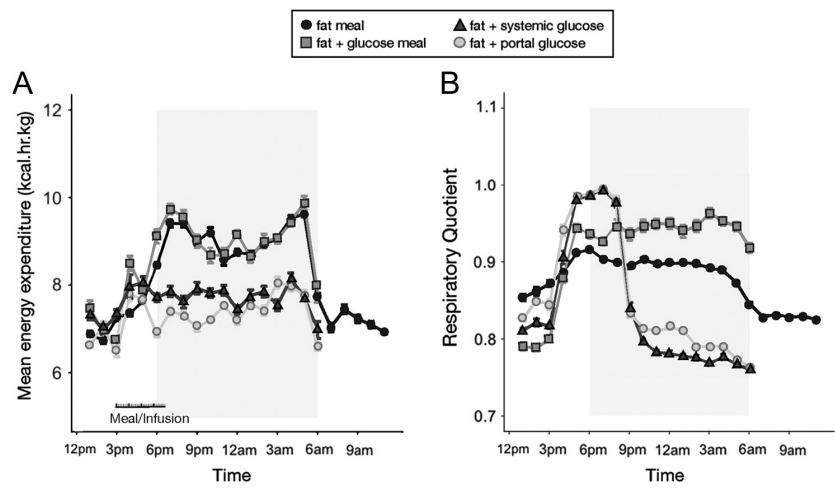

Figure 7

(A) Energy expenditure and (B) respiratory quotient over $24 \mathrm{~h}$ prior to clamp experiments in rats fed either a high-fat diet $(n=6)$, high-fat diet + high-glucose meal $(n=3)$, high-fat diet + systemic glucose infusion $(n=4)$ or high-fat diet + portal glucose infusion $(n=3)$. Meals and glucose infusions were given at 15:00 h. Dark grey shading indicates dark phase of light cycle. Data are mean \pm S.E.M.

(c) 2019 Society for Endocrinology Published by Bioscientifica Ltd. Printed in Great Britain muscle mitochondria from 12-week high-fat-fed rats has been reported (Jorgensen et al. 2017).

Metabolic adaptations to acute changes in diet and specific macronutrients have not been well characterised, even though there is substantial literature on changes in metabolic pathways and enzyme levels in response to fasting and refeeding (Korbonits et al. 2007). Han et al. (2009) measured insulin-stimulated glucose uptake in isolated skeletal muscles from chow and high-fat-fed rats fed a glucose meal and demonstrated significant increases in response to the glucose meal. This validates the phenomenon we observed and provides evidence that the improvement in insulin action is not a direct reversal of the processes driving insulin resistance induced by high-fat feeding. Rather, a different mechanism that is able to enhance insulinstimulated glucose disposal is responsible and a role for portal glucose sensing is very plausible. Because portal glucose infusion was also successful in improving insulin action, this negates the role of nutrient-sensing mechanisms in the gut, incretins such as GLP-1, GIP and the microbiota, all of which are well documented 
to alter glucose metabolism (Drucker 2007, Khan et al. 2014, Kootte et al. 2017).

Future studies should explore the mechanism of how the hepatoportal region mediates this improvement. The vagal nerve can detect portal glucose levels and signal via the central nervous system to regulate whole body metabolism (Trung et al. 2014). Determining the effect of a glucose meal or portal glucose infusion on insulin sensitivity in high-fat-fed vagotomised rats will provide insight into the role of the vagal nerve in this model and identify if the central nervous system plays a key role in the observed improvement.

This study highlights the acute and adaptive changes in metabolism that are possible as a result of alterations in macronutrient composition. Whether or not this system is relevant in regulating human insulin sensitivity is an important question for future studies. Insulin sensitivity is very likely modulated by diet, and our results help clarify the acute effects of diet on the regulation of insulin action and point to potential mechanisms of macronutrient sensing in the hepatoportal region that may be important for this.

\section{Declaration of interest}

The authors declare that there is no conflict of interest that could be perceived as prejudicing the impartiality of the research reported.

\section{Funding}

This research did not receive any specific grant from any funding agency in the public, commercial or not-for-profit sector.

\section{Author contribution statement}

$H M J, E S, G J C^{1}, E E E, G J C^{2}$ and $K S$ B-A performed the experiments. $\mathrm{G} J \mathrm{C}^{2}$ and $\mathrm{K} \mathrm{S}$ B-A designed the experiments. $\mathrm{H} \mathrm{M} \mathrm{J,} \mathrm{G} \mathrm{J} \mathrm{C1,} \mathrm{G} \mathrm{J} \mathrm{C}^{2}$ and K S B-A wrote the paper.

\section{References}

Bernard C 1877 Leçons sur le diabète et la glycogenèse animale. Paris, France: J-B Baillière et Fils.

Berthoud HR, Kressel M \& Neuhuber WL 1992 An anterograde tracing study of the vagal innervation of rat liver, portal vein and biliary system. Anatomy and Embryology 186 431-442. (https://doi. org/10.1007/BF00185458)

Bohland M, Matveyenko AV, Saberi M, Khan AM, Watts AG \& Donovan CM 2014 Activation of hindbrain neurons is mediated by portal-mesenteric vein glucosensors during slow-onset hypoglycemia. Diabetes 63 2866-2875. (https://doi.org/10.2337/db13-1600)

Buettner R, Parhofer KG, Woenckhaus M, Wrede CE, Kunz-Schughart LA, Scholmerich J \& Bollheimer LC 2006 Defining high-fat-diet rat models: metabolic and molecular effects of different fat types. Journal of Molecular Endocrinology 36 485-501. (https://doi.org/10.1677/jme.1.01909)
Burcelin R, Dolci W \& Thorens B 2000 Portal glucose infusion in the mouse induces hypoglycemia: evidence that the hepatoportal glucose sensor stimulates glucose utilization. Diabetes 49 1635-1642. (https:// doi.org/10.2337/diabetes.49.10.1635)

Coate KC, Kraft G, Irimia JM, Smith MS, Farmer B, Neal DW, Roach PJ, Shiota M \& Cherrington AD 2013 Portal vein glucose entry triggers a coordinated cellular response that potentiates hepatic glucose uptake and storage in normal but not high-fat/high-fructose-fed dogs. Diabetes 62 392-400. (https://doi.org/10.2337/db12-0417)

Daly ME, Vale C, Walker M, Alberti KG \& Mathers JC 1997 Dietary carbohydrates and insulin sensitivity: a review of the evidence and clinical implications. American Journal of Clinical Nutrition 66 1072-1085. (https://doi.org/10.1093/ajcn/66.5.1072)

Drucker DJ 2007 The role of gut hormones in glucose homeostasis. Journal of Clinical Investigation 117 24-32. (https://doi.org/10.1172/ JCI30076)

Feinman RD, Pogozelski WK, Astrup A, Bernstein RK, Fine EJ, Westman EC, Accurso A, Frassetto L, Gower BA, McFarlane SI, et al. 2015 Dietary carbohydrate restriction as the first approach in diabetes management: critical review and evidence base. Nutrition 31 1-13. (https://doi.org/10.1016/j.nut.2014.06.011)

Goodpaster BH \& Sparks LM 2017 Metabolic flexibility in health and disease. Cell Metabolism 25 1027-1036. (https://doi.org/10.1016/j. cmet.2017.04.015)

Han DH, Hancock C, Jung SR \& Holloszy JO 2009 Is "fat-induced" muscle insulin resistance rapidly reversible? American Journal of Physiology. Endocrinology and Metabolism 297 E236-E241. (https://doi. org/10.1152/ajpendo.00244.2009)

Hernandez EÁ, Kahl S, Seelig A, Begovatz P, Irmler M, Kupriyanova Y, Nowotny B, Nowotny P, Herder C, Barosa C, et al. 2017 Acute dietary fat intake initiates alterations in energy metabolism and insulin resistance. Journal of Clinical Investigation 127 695-708. (https://doi. org/10.1172/JCI89444)

International Diabetes Federation 2013 IDF Diabetes Atlas, 6th edn Brussels, Belgium: International Diabetes Federation.

Jorgensen W, Rud KA, Mortensen OH, Frandsen L, Grunnet N \& Quistorff B 2017 Your mitochondria are what you eat: a high-fat or a high-sucrose diet eliminates metabolic flexibility in isolated mitochondria from rat skeletal muscle. Physiological Reports 5 e13207. (https://doi.org/10.14814/phy2.13207)

Khan MT, Nieuwdorp M \& Backhed F 2014 Microbial modulation of insulin sensitivity. Cell Metabolism 20 753-760. (https://doi. org/10.1016/j.cmet.2014.07.006)

Kootte RS, Levin E, Salojarvi J, Smits LP, Hartstra AV, Udayappan SD, Hermes G, Bouter KE, Koopen AM, Holst JJ, et al. 2017 Improvement of insulin sensitivity after lean donor feces in metabolic syndrome is driven by baseline intestinal microbiota composition. Cell Metabolism 26 611.e6-619.e6. (https://doi.org/10.1016/j.cmet.2017.09.008)

Korbonits M, Blaine D, Elia M \& Powell-Tuck J 2007 Metabolic and hormonal changes during the refeeding period of prolonged fasting. European Journal of Endocrinology 157 157-166. (https://doi. org/10.1530/EJE-06-0740)

Kraegen EW, James DE, Jenkins AB \& Chisholm DJ 1985 Dose-response curves for in vivo insulin sensitivity in individual tissues in rats. American Journal of Physiology 248 E353-E362. (https://doi. org/10.1152/ajpendo.1985.248.3.E353)

McClenaghan NH 2005 Determining the relationship between dietary carbohydrate intake and insulin resistance. Nutrition Research Reviews 18 222-240. (https://doi.org/10.1079/NRR2005109)

Oakes ND, Bell KS, Furler SM, Camilleri S, Saha AK, Ruderman NB, Chisholm DJ \& Kraegen EW 1997 Diet-induced muscle insulin resistance in rats is ameliorated by acute dietary lipid withdrawal or a single bout of exercise: parallel relationship between insulin stimulation of glucose uptake and suppression of long-chain fatty acyl-CoA. Diabetes 46 2022-2028. (https://doi.org/10.2337/ diab.46.12.2022) 
Parillo M \& Riccardi G 2004 Diet composition and the risk of type 2 diabetes: epidemiological and clinical evidence. British Journal of Nutrition 92 7-19. (https://doi.org/10.1079/BJN20041117)

R Core Team 2016 R: A Language and Environment for Statistical Computing. Vienna, Austria: R Foundation for Statistical Computing.

Small L, Brandon AE, Turner N \& Cooney GJ 2018 Modeling insulin resistance in rodents by alterations in diet: what have high-fat and highcalorie diets revealed? American Journal of Physiology. Endocrinology and Metabolism 314314 E251. (https://doi.org/10.1152/ajpendo.00337.2017)

Storlien LH, Kraegen EW, Chisholm DJ, Ford GL, Bruce DG \& Pascoe WS 1987 Fish oil prevents insulin resistance induced by high-fat feeding in rats. Science 237 885-888. (https://doi.org/10.1126/science.3303333)

Sweeney JS 1927 Dietary factors that influence the dextrose tolerance test. Archives of Internal Medicine 40 818-830. (https://doi.org/10.1001/arch inte.1927.00130120077005)
Trung VN, Yamamoto H, Yamaguchi T, Murata S, Aimi Y, Kuwahara A \& Tani T 2014 Intact neural system of the portal vein is important for maintaining normal glucose metabolism by regulating glucagon-like peptide-1 and insulin sensitivity. Peptides 52 38-43. (https://doi. org/10.1016/j.peptides.2013.12.003)

Turner N, Kowalski GM, Leslie SJ, Risis S, Yang C, Lee-Young RS, Babb JR, Meikle PJ, Lancaster GI, Henstridge DC, et al. 2013 Distinct patterns of tissue-specific lipid accumulation during the induction of insulin resistance in mice by high-fat feeding. Diabetologia 56 1638-1648. (https://doi.org/10.1007/s00125013-2913-1)

Vella A \& Rizza RA 2009 Application of isotopic techniques using constant specific activity or enrichment to the study of carbohydrate metabolism. Diabetes 58 2168-2174. (https://doi. org/10.2337/db09-0318)

Received in final form 27 February 2019

Accepted 2 April 2019

Accepted Preprint published online 2 April 2019 (c) 2019 Society for Endocrinology Published by Bioscientifica Ltd.
Printed in Great Britain 